$32^{\text {nd }}$ European Modeling \& Simulation Symposium

$17^{\text {th }}$ International Multidisciplinary Modeling \& Simulation Multiconference

ISSN 2724-0029 ISBN 978-88-85741-44-7 (c) 2020 The Authors.

doi: $10.46354 /$ i3m.2020.emss.062

\title{
Extended Reality Technologies for Industrial Innovation
}

\author{
Agostino G. Bruzzone ${ }^{*}$, Kirill Sinelshchikov ${ }^{2}$, Marina Massei ${ }^{1}$, Giuliano \\ Fabbrini $^{2}$, Marco Gotelli ${ }^{2}$ \\ ${ }^{1}$ Simulation Team, SIM4Future, via Trento 34, 16145 Genova, Italy \\ ${ }^{2}$ Simulation Team, DIME, University of Genova, via Opera Pia 15, 16145 Genova, Italy \\ *Corresponding author. Email address: agostino.bruzzone@sim4future.com
}

\begin{abstract}
This paper presents an overview on immersive technologies in relation to their potential for industrial innovation. In particular, Extended Reality (XR) is proposed by describing the most common solutions and innovative methods to overcome inherent problems of these technologies. Virtual \& Augmented Reality (VR \& AR ) are presented respect their potential for uses such as innovative human-machine interfaces, remote maintenance, remote commissioning, 3D rendering, virtual factory, virtual assembly and training. The paper analyze the strong contribution that Immersive Technologies are bringing in multiple sectors including Industries and the future trends aiming to further increase usability of these technologies such as it is happening in overcoming spatial constraints.
\end{abstract}

Keywords: Virtual Reality; Augmented Reality; Extended Reality; Free-roaming

\section{Introduction}

Improving processes with immersive technologies is an opportunity that companies, characterized by complex production processes and product development, are taking advantage of (Berg \& Vance, 2017). Nowadays, software and hardware developments have been greatly fast, allowing high technology to be deployed in the market for significantly beneficial use cases. Nowadays, Immersive Technologies, such as Extended Reality (XR), Mixed Reality (MR), Virtual Reality (VR), Augmented Reality AR), are creating new opportunities. Indeed the Immersive Technologies, also known as Digital Reality, jointly with the Internet of Things (IoT) and complex control systems are paving the way for huge innovations. Digital Reality allows users to interact with a virtual environment in an apparently physical way using wearable (i.e. HMD) or mobile devices. Currently there are some significant use cases in the industrial sector regarding several topics: remote collaboration, immersive and collaborative training, data visualization and design. The benefits affect the whole process starting from the enhancement of the product design to the improvement of proficiency, supply chain planning, and workforce collaboration. These have had a real boost in the last years solving some of the inherent problems of these technologies. Headsets are especially experiencing great innovations. VR devices are decreasing the image latency at a high rate, the enhancement of mobile computing will soon eliminate the need to connect and new breakthroughs in tracking objects, body parts and movements are opening the way for a whole set of applications.

\section{Existing Technologies and Future Trends}

The technologies used to create an immersive

(C) 2020 The Authors. This article is an open access article distributed under the terms and conditions of the Creative Commons Attribution (CC BY-NC-ND) license (https://creativecommons.org/licenses/by-nc-nd/4.0/). 
experience are becoming more and more sophisticated and realistic, while at the same time their cost are downgraded making it possible to extend their use in many more application areas (Hale \& Stanney, 2014; Bruzzone et al.,2016a; Berg \& Vance, 2017). Along last years we moved from original 1 million USD cost of a CAVE (Cave Automatic Virtual Environment) around 20 years ago, to 40'000 USD for the basic configuration of a modern compact interoperable and touchscreen system such as SPIDER (Simulation Practical Immersive Dynamic Environment for Reengineering) introduced by Simulation Team (Hereld et al., 2000; Cruz-Neira et al.,2013; Bruzzone et al.,2016b). What it is interesting in relation to SPIDER is that even if visualization just FULL HD over $2 \mathrm{~m}$ wide screens surrounding the people inside the cube, it introduces two very innovative concept at low price:

- touch screen solution that enables to touch the screens all around SPIDER users (in the above mentioned basic configuration over $270^{\circ}$ horizontally within the cubicle of $2 \mathrm{~m} \times 2 \mathrm{~m} \times 2.6 \mathrm{~m}$ ) and interact with objects

- fully interoperability allowing to combine the SPIDER with multiple models and simulators as part of a Federation

This means that even low cost solutions nowadays introduce very high value new features and capabilities respect old classical expensive solutions that were limiting the use mostly just to defense and aerospace (Vandervliet 1992; Oberhauser et al.,2015)

In addition, the developments in the field of VR \& AR devices have create a scalable set of solutions that moves from 400'000 USD of an advanced Visualization System to 40'000 of a SPIDER, 4'000 for AR with Hololens, 400 for VR with Oculus Rift down to less than 40 Euro for a Headset holder for a Smartphone able to support stereoscopic viewing and VR (Papachristos et al.,2017; Bruzzone et al. 2019; Elor et al.2020). Obviously in case of distributed interactive simulation it is crucial proper adoption of correct protocols and data exchange as well as techniques to improve connectivity performance (Bruzzone et al., 1998, Zeigler et al., 1998; Fujimoto 2000; 2017; Liu et al., 2014; Graf et al., 2017).

So today, there are many mix of solutions that, based on the different application and user, results to be the most effective.

\subsection{Overview on Existing Technologies}

Virtual Reality and Simulation have a very consolidated history, therefore it is fundamental to outline that current solutions are turned to be much more effective and efficient of past ones with limited costs as already stated before, so following survey provides an overview on the major components of modern XR solutions.

\subsubsection{Systems \& Devices}

Smartphones and tablets are the simplest device to be used due to their wide adoption. By the way, it is possible to create valuable experiences when they do not need a high level of complexity in operations and graphics (Steed \& Julier, 2013; Ko et al.,2013).

These devices are mostly used for augmented reality. Augmented Reality (AR) overlays virtual and real world. For example, it could introduce text, images and animations on top of physical objects. Mixed Reality (MR) term is used to describe more interactive AR (Benford \& Gianmachi, 2011).

Interesting use cases for $\mathrm{AR}$ are the ones where operators and experts are geographically spread (i.e. Remote Maintenance, remote commissioning, etc.). Today VR and AR could provide major benefits in remote service and maintenance as demonstrated by recent researches by the authors (Bruzzone et al.,2016d). Indeed, developing a web application based on mobile devices that tracks objects and overlap virtual ones can be a competitive advantage, allowing companies and agencies to reap a huge amount of money and improve communication in tough situations (Bruzzone et al.,2016a; Mourtzis et al.2017).

For Virtual Reality the set of equipment includes, but not limited to Head Mounted Displays (HMD), projected environments, controllers, motion platforms, motion tracking systems, haptic gloves and suits. Principal fields of application of VR are education, training and entertainment, but also support of engineering and safety preparation (Jacobson \& Hwang 2002; Hale \& Stanney, 2014); by the way recently even autonomous systems are turning in additional elements of modern XR for multiple purposes (Bruzzone et al., 2016c; Kim et al., 2018). However, technologies and objectives are evolving and changing rapidly (Muñoz-Saavedra et al.,2020).

VR solutions are used for training of operators of different kind of machinery in industry (e.g. cranes), facilities (e.g. industrial plants) as well as for recreational (e.g. museums \& exhibitions) \& educational purposes in classrooms (Whisker et al., 2003; Bruzzone \& Longo, 2013; Mastli \& Zhang, 2017; Longo et al.,2018; Liu et al.,2020; Puig et al., 2020).

Most products include not only headsets, but also controllers, capable to provide interaction capabilities with the virtual world (Sagayam \& Hemanth, 2017; Kumari \& Polke, 2018). For example, many controllers allow tracking not only position but also spatial orientation, which can be used to 'point' on virtual objects, drag and drop them. Such systems can include different set of inputs, starting from one simple click button and up to combination of joysticks, buttons and touch sensors capable to detect "near click" (Bowman et al., 2012; Coelho et al., 2014).

There are solutions that are also non wearable. The most common are called CAVES (Cruz Neira et al., 
2012). CAVES have been used in many different fields, from military training to medicine to visualize parts of a body giving so an opportunity to prepare for operation in shared environment (Hale et al. 2014). In addition to these fields there are CAVE used in Universities and Industries as virtual show rooms, or in Museum for the reproduction of natural or past environment (Muhanna, 2015). In facts, some applications of CAVE are specifically related to entertainment sector (Jacobson \&amp; Hwang 2002). In facts, a CAVE contains usually a limited space where virtual world is reproduced, but it allow the users to enter and eventually, by most modern solutions, to interact with it (Hale et al. 2014; Bruzzone et al. 2016a). Images could be created using classic direct view otherwise rear projection, which reduce drastically number of components inside CAVE, hence improving its virtual immersion, however this solution is not so comfortable in exploitation and requires bigger external volume and space occupancy of the whole equipment (Hale et al. 2014.

\subsubsection{Motion Platform}

The user experience of both the HMD and Caves can be enhanced through the integration of a motion platform (Advani \& Va, 1995; Chen 2001; Thöndel 2010; Lee 2020). They allow simulation of vibration, collisions and accelerations. It is possible to employ in virtual reality even more specific or specialized equipment (VanderVliet 1992; Pollini et al.,2008). In practice, nowadays more and more devices are being developed with VR integration in mind. In the following lines is shown a specific motion platform that has been used for helicopter simulation that represent an interesting application field (Schroeder, 1999).

Figure 1 proposes as example a 6 DOF (Degrees of Freedom) motion device based on Steward Platform (in this case courtesy of Brunner for model Motion1000); the corresponding motion control system provides different motion cueing algorithms, allowing adapt the device for different types of simulations. A motion platform represent an interesting opportunity for improving fidelity of the flight simulator and it reduces discrepancies between the vestibular and the visual system during the virtual reality simulation; therefore in flight-simulators this is on the most sickening factors. It is important to outline that Artificial Intelligence has a great potential in reducing the impact of motion sickness by developing innovative control systems able to mitigate it though prediction of users movements (Hell et al., 2018). Therefore it is always necessary to remember that is fundamental to properly evaluate the advantage provided by motion platform respect the specific objective of the simulator (Amico et al.,2000).

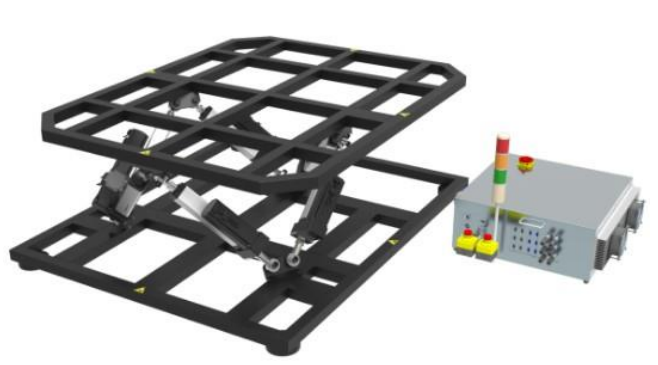

Figure 1. Steward Motion Platform 6 DoF

\subsubsection{Tracking Systems}

The applications that imply a physical movement of one or more users need a tracking system (Chang et al., 2001; Cameron et al.,2011; Shin et al.,2016; Park et al., 2017). As aforementioned, HMDs have their own tracking system for a narrow area. For custom applications or for expanding the available walking area there are different technologies for navigation and positioning.

In the following lines, different type of devices will be analyzed:

- Inertial measurements units (IMU): They are devices capable to detect angular and linear accelerations (Zhang et al., 2015). These sensors are usually relatively cheap and allow to provide minimum positioning to various devices, such as smartphones. These systems do not depend on external services or infrastructures. IMUs are particularly useful for dead reckoning - to guarantee positioning even after external connections are lost (e.g. GPS after enter in tunnel). The principal component of any IMU are accelerometers and gyroscopes. It possible to find different types of accelerometers and gyroscopes , for instances in mobile systems they are very small, yet they provide high level of precision

- GNSS: One of most known systems for outdoor positioning is GPS, which is used in most of modern mobile phones. It allows to identify coordinates in most of the world, even if final precision could be not sufficient for certain purposes. There are several projects which employ positioning data obtained from GNSS and gyroscopes in augmented reality applications, for instance, to extend functionality of navigators (Angelino et al.,2012; Wang et al.,2012).

- Radio beacons: In some situations it is convenient to utilize general purpose indoor positioning systems (Foxlin et al.,1998); for instance, they often offer higher range of operations respect custom solutions. Currently, several different technologies are used for indoor positioning, most diffused are:

1. Bluetooth 


\section{UWB \\ 3. Wifi}

- Constellation tracking: It is inspired by the old method navigation (Cortez et al.,2017). Indeed, each device is equipped with unique configuration of IR LEDs, visible only to tracking stations, which are capable to individuate position and orientation of each piece of equipment. This type of tracking requires brief installation and configuration of equipment following interactive instruction. Positioning is based on external sensors so proper positioning is require.

Focusing on Augmented reality, modern toolkits allow using various objects as positing tags: QR codes, images, surfaces with patterns (Pence, 2010). Tag recognition starts by detecting "features" like sharp or spiked details image.

It is highly recommended that such tags have high contrast, elevated number of features and avoid too repetitive patterns (e.g. grids). The disadvantage of this approach is that false positive and false negative detection has relatively high probability and that image targets must satisfy basic requirements.

In virtual reality headset, the tracking system continuously maps the environment around and tries to correlate it with known ones, consequently understanding its relative position.

From the experience in industrial projects in emerged a difficulty in operation in following conditions:

- Presence of multiple moving obstacles which obstruct positioning, such as human operators or conveyor belts

- Operation in zones with wide empty areas, for example free part of a warehouse and very big rooms, which overwhelm capacity of recognition of $3 \mathrm{D}$ vision and depth perception sensors

Tracking users' movements enhance these applications also for motion capture. Motion capture data in immersive experiences might be used both to register real user behaviors and reproduce them in avatars and to build predictive models. Indeed, predictive algorithms applied to tracking systems can improve the system performance forecasting in realtime which will be the users movements during the experience. Some examples of motion capture are:

- Mechanical or magnetic: Physical sensors are attached to the body.

- Optical-passive: Is based on reflective markers tracked by cameras, usually operating in IR. High precision, widely used.

- Optical-active: Similar to the previous one but uses LED markers.
- Video marker less: Software-based tracking of objects in video feed.

- Inertial: Position of objects is dead reckoned by IMU

It is particularly challenging to track in high fidelity the hands movements. Controllers provide a basic tracking. For example the HMDs controllers can detect whether a hand is open or not and basic movements of fingers. A technological solution that is being widely is the Leap Motion is an optical hand-tracking device that captures the movement hands. The Leap Motion is mounted on the front part of HMDs; this causes the main shortfall of the device. Indeed, the leap motion tracks the movement only when the user is looking at their hands. By the way, it constitutes a cost effective and precise solution.

\subsection{Overview on Future Trends}

The immersive technologies have a great development both in technology and applications and provide even new ways to create special experience for users affecting their perception and emotions in a more deep way (Gonzalez et al., 2015). Hereby are listed some trends that could a breakthrough in this field:

- Retinal Projection

- Free-Roaming

- Digital Twin Integration

- New Connectivity

\subsubsection{Retinal Projection}

Recently there is a growing interest to retinal display technology, which project image directly into eye retina (Kenyon et al., 2014; Mi et al., 2018). Example of such recent project is Intel Vaunt, which look like normal glasses from the outside.

\subsubsection{Free-Roaming}

Free-roaming allows trainees/users to move freely within a room by tracking their movements (Wang et al.,2011). In fact, the system reproduces exactly the movements in a parallel virtual environment that remains aligned to the action of the people involved and any virtual twin of physical objects, whether real or mockup; this allows users to interact with each other and with the simulation. These systems can work in an integrated way with Virtual reality using free roaming as an added value to experiment different scenarios for training or decision support. It is possible to train trainees to respond to sudden events or perform maintenance operations of complex equipment or damaged vehicles in a virtual environment.

The possibility of interaction between one trainees/user and the other is a breakthrough, combining Virtual Reality (Free Roaming compared to 
common Virtual Reality systems).

Below are listed the main advantages of this approach:

- Tracking of body movements including upper and lower limbs and fingers. (Ex: The trainee has the ability to follow the movements of his hands within the virtual environment)

- Social Interaction of Trainees: Trainees can cooperate thanks to the creation of avatars to which real movements are associated, tracked by trackers.

- Interaction with virtual objects: the same trackers can also be placed on physical devices, associating them to virtual objects that are reported within the scenario

- Modularity: the system can manage from 1 to 6 trainees

- Space Scalability: You can develop flexible applications operating on different scenarios and sizes. In the following the technical description is based on a reference size of $25 \mathrm{~m} 2$, but of course it is possible to extend and scale the system for larger spaces with additional devices and upgrades.

- Retention Rate: Through the use of virtual reality with free-roaming is guaranteed a faster learning compared to traditional technologies. In how much it is tied up the training to particularly immersive experiences, where the proprioceptive elements and of psicophysical fellowship are emphasized from the tracking of the behavior of the subjects and their body during the simulated activities.

- Overcoming spatial constraints: The key point of the innovativeness of the system lies in the fact that the user, or trainee, will not have the constraints of movement imposed by traditional VR systems, as the free-roaming system is Wireless and, through tracking, allows you to walk, move, climb on objects, move between different rooms allowing, possibly, the extension on articulated spaces (e.g. inside a building, plant or vessel).

- Transportable: the system can be easily disassembled and assembled to be moved according to needs.

- Detection of biometric parameters (e.g. muscle tone, heartbeat) through an optional module that can be integrated.

The movements need to be tracked and the data have to be sent to the local computer. In order to do so, it is proposed the following scheme:

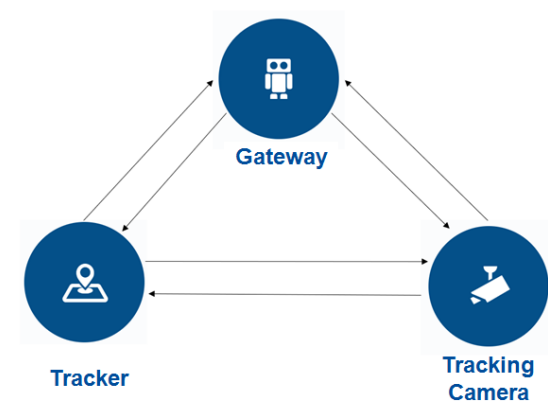

Figure 2 - Tracking

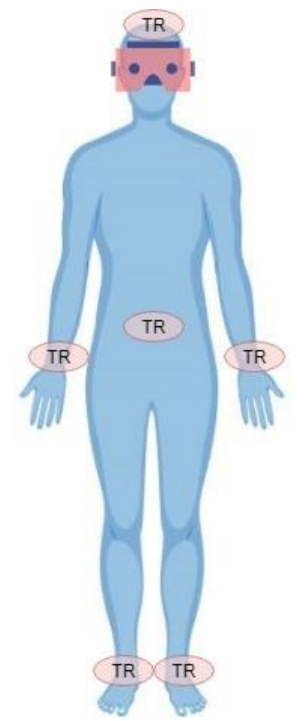

Figure 3 - Body Trackers

The tracking camera follow the tracker mounted on the users like in Figure 3. An example of the tracking cameras for a default space of 25 square meters is shown in figure 4 .

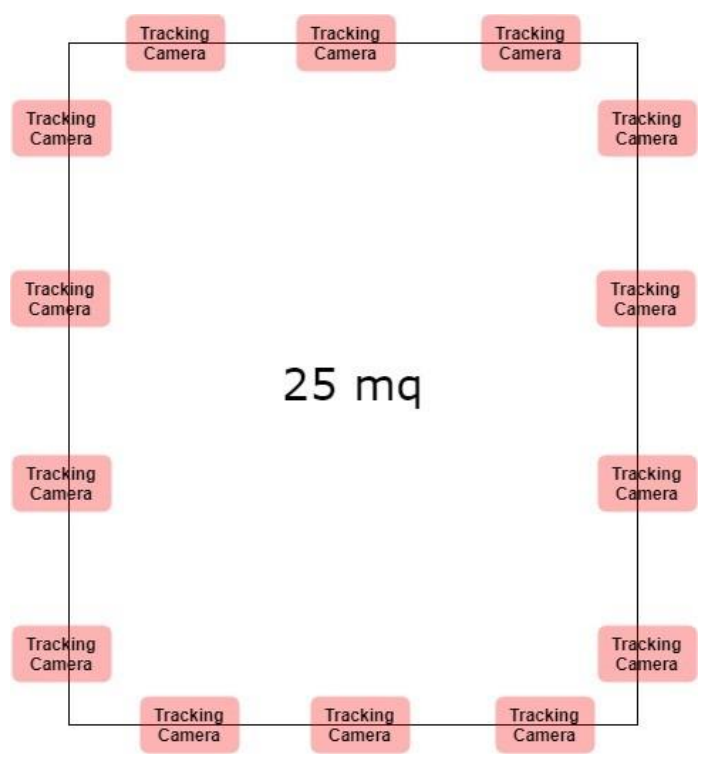

Figure 4 - Tracking Camera 


\subsubsection{Digital Twin Integration}

Digital twin is a term that has been used firstly in 2002, but it became actual during the recent years due to the advent on low cost sensors (Qi \& Tao, 2018). Indeed, with WSN (wireless sensor network) it is possible to create a real time data stream of parameters that describe the behavior of physical objects. Showing relevant data during the production is indeed an important aspect in production. For instance, there are some use cases of visualizing data overlapped to high fidelity models of complex systems (production lines, turbines, harbor security, etc.) and visualizing key information directly on site ( $\mathrm{Lu} \& \mathrm{Xu}$, 2018). Another aspect is about the integration of immersive scenarios on product lifecycle management tools.

\subsubsection{Digital Twin Integration}

The connectivity speed and reliability is fast increasing and its turning to be even more accessible due to emerging technologies; so it is evident the potential benefits for Extended Reality in drastical reduction of latency by new technological solutions such as 5G (Gomez-Barquero et al., 2019)

\section{Conclusions}

This study provides an overview on several solutions devoted to apply Extended to a wide spectrum of applications. In fact the immersive technologies demonstrate along last three decades an impressive evolution and are moving out of research labs into industries as well as in common our life . Current emerging trends and future research are directed towards new concepts that stress the interoperability and intuitiveness required to make these solutions even more usable. For sure, Artificial Intelligence is a major driver in this sense considering that is able to extend the capabilities of these systems and integrate dynamically data in consistently way

\section{References}

Advani, S., \& Va, M. (1995). The design of a highperformance all-composite flight simulator motion platform. In Flight Simulation Technologies Conference (p. 3382).

Amico, V., Bruzzone, A. G., \& Guha, R. (2000, July). Critical issues in simulation. In SUMMER COMPUTER SIMULATION CONFERENCE (pp. 893898). Society for Computer Simulation International; 1998.

Angelino, C. V., Baraniello, V. R., \& Cicala, L. (2012, July). UAV position and attitude estimation using IMU, GNSS and camera. In 2012 15th International Conference on Information Fusion (pp. 735-742). IEEE.

Benford, S., \& Giannachi, G. (2011). Performing mixed reality. The MIT Press.
Berg, L. P., \& Vance, J. M. (2017). Industry use of virtual reality in product design and manufacturing: a survey. Virtual reality, 21(1), 1-17.

Bowman, D. A., McMahan, R. P., \& Ragan, E. D. (2012). Questioning naturalism in 3D user interfaces. Communications of the ACM, 55(9), 78-88.

Brunner Elektronik AG, Factsheet 6DOF Motion 1000, Industriestrasse 27, $\mathrm{CH}-8335$ Hittnau

Bruzzone, A. G., \& Giribone, P. (1998, April). Decisionsupport systems and simulation for logistics: Moving forward for a distributed, real-time, interactive simulation environment. In Proceedings 31st Annual Simulation Symposium (pp. 17-24). IEEE.

Bruzzone, A. G., \& Longo, F. (2013). 3D simulation as training tool in container terminals: The TRAINPORTS simulator. Journal of Manufacturing Systems, 32(1), 85-98.

Bruzzone, A. G., \& Massei, M. (2017). Simulationbased military training. In Guide to SimulationBased Disciplines (pp. 315-361). Springer, Cham.

Bruzzone, A. G., Fancello, G., Daga, M., Leban, B., \& Massei, M. (2019). Mixed reality for industrial applications: interactions in human-machine system and modelling in immersive virtual environment. International Journal of Simulation and Process Modelling, 14(2), 165-177.

Bruzzone, A. G., Longo F., Nicoletti L., Vetrano M., Bruno L., Chiurco A., Fusto C., Vignali G. (2016d). Augmented reality and mobile technologies for maintenance, security and operations in industrial facilities. 28th European Modeling and Simulation Symposium, EMSS 2016, pp. 355

Bruzzone, A. G., Longo, F., Agresta, M., Di Matteo, R., \& Maglione, G. L. (2016b). Autonomous systems for operations in critical environments. In Proceedings of the Modeling and Simulation of Complexity in Intelligent, Adaptive and Autonomous Systems 2016 (MSCIAAS 2016) and Space Simulation for Planetary Space Exploration (SPACE 2016) (pp. 18).

Bruzzone, A. G., Massei, M., Maglione, G. L., Agresta, M., Franzinetti, G., \& Padovano, A. (2016a). Virtual and augmented reality as enablers for improving the service on distributed assets. Proc. of I3M, Larnaca, Cyprus, September.

Bruzzone, A. G., Massei, M., Maglione, G. L., Di Matteo, R., \& Franzinetti, G. (2016c). Simulation of manned \& autonomous systems for critical infrastructure protection. Proceedings of DHSS, Larnaca, Cypurs, September.

Cameron, C. R., DiValentin, L. W., Manaktala, R., McElhaney, A. C., Nostrand, C. H., Quinlan, O. J., ... \& Gerling, G. J. (2011, April). Hand tracking and visualization in a virtual reality simulation. In 2011 IEEE systems and information engineering design 
symposium (pp. 127-132). IEEE.

Chang, C. C., \& Tsai, W. H. (2001). Vision-based tracking and interpretation of human leg movement for virtual reality applications. IEEE transactions on circuits and systems for video technology, 11(1), 9-24.

Chen, J., Fang, Y. C., Loftin, R. B., Leiss, E. L., Lin, C. Y., \& Su, S. (2001). An immersive virtual environment training system on real-time motion platform. Proc. of the Computer Aided Design and Computer Graphics, 2, 951-954.

Coelho, J. C., \& Verbeek, F. J. (2014). Pointing task evaluation of leap motion controller in 3D virtual environment. Creating the difference, 78, 78-85.

Cortes, G., Marchand, E., Ardouinz, J., \& Lécuyer, A. (2017, March). Increasing optical tracking workspace of VR applications using controlled cameras. In 2017 IEEE Symposium on 3D User Interfaces (3DUI) (pp. 22-25). IEEE.

Cruz-Neira, C., Sandin, D. J., \& DeFanti, T. A. (1993, September). Surround-screen projection-based virtual reality: the design and implementation of the CAVE. In Proceedings of the 2oth annual conference on Computer graphics and interactive techniques (pp. 135-142).

Cruz-Neira, C., Sandin, D.J., DeFanti, T.A., Kenyon, R.V., Hart, J.C. (1992) "The CAVE: audio visual experience automatic virtual environment", ACM Commun., 35 (6), 64-72.

Elor, A., Powell, M., Mahmoodi, E., Hawthorne, N., Teodorescu, M., \& Kurniawan, S. (2020). On shooting stars: Comparing cave and hmd immersive virtual reality exergaming for adults with mixed ability. ACM Transactions on Computing for Healthcare, 1(4), 1-22.

Foxlin, E., Harrington, M., \& Pfeifer, G. (1998, July). Constellation: a wide-range wireless motiontracking system for augmented reality and virtual set applications. In Proceedings of the 25th annual conference on Computer graphics and interactive techniques (pp. 371-378).

Fujimoto, R. M. (2000). Parallel and distributed simulation systems (Vol. 300). New York: Wiley.

Gomez-Barquero, D., Li, W., Fuentes, M., Xiong, J., Araniti, G., Akamine, C., \& Wang, J. (2019). IEEE Transactions on Broadcasting special issue on: 5G for broadband multimedia systems and broadcasting. IEEE Transactions on Broadcasting, 65(2), 351-355.

Gonzalez, D. S., Moro, A. D., Quintero, C., \& Sarmiento, W. J. (2016, August). Fear levels in virtual environments, an approach to detection and experimental user stimuli sensation", Proc. of XXI IEEE Symposium on Signal Processing, Images and Artificial Vision (STSIVA), pp. 1-6
Graf, M., Timmerer, C., \& Mueller, C. (2017, June). Towards bandwidth efficient adaptive streaming of omnidirectional video over http: Design, implementation, and evaluation. In Proceedings of the 8th ACM on Multimedia Systems Conference (pp. 261-271).

Hale, K. S., \& Stanney, K. M. (2014). "Handbook of virtual environments", CRC Press, Boca Raton, FL

Hell, S., \& Argyriou, V. (2018, December). Machine learning architectures to predict motion sickness using a virtual reality rollercoaster simulation tool. In 2018 IEEE International Conference on Artificial Intelligence and Virtual Reality (AIVR) (pp. 153156). IEEE.

Hereld, M., Judson, I. R., \& Stevens, R. L. (2000). "Introduction to building projection-based tiled display systems". IEEE Computer Graphics and Applications, 20. pp. 22-28

Jacobson J.,Hwang Z.(2002) "Unreal tournament for immersive interactive theater", ACM Comm., 45 (1), 39-42

Kenyon, A., Van Rosendale, J., Fulcomer, S., \& Laidlaw, D. (2014). "The design of a retinal resolution fully immersive VR display", Virtual Reality (VR), IEEE, pp. 89-90

Kim, S. J., Jeong, Y., Park, S., Ryu, K., \& Oh, G. (2018). A survey of drone use for entertainment and AVR (augmented and virtual reality). In Augmented Reality and Virtual Reality (pp. 339-352). Springer, Cham.

Ko, S. M., Chang, W. S., \& Ji, Y. G. (2013). Usability principles for augmented reality applications in a smartphone environment. International journal of human-computer interaction, 29(8), 501-515.

Kumari, S., \& Polke, N. (2018, August). Implementation Issues of Augmented Reality and Virtual Reality: A Survey. In International Conference on Intelligent Data Communication Technologies and Internet of Things (pp. 853-861). Springer, Cham.

Lee, S. M., Xia, K., \& Son, H. (2020). Robust Tracking Control of Spherical Motion Platform for Virtual Reality. IEEE Transactions on Industrial Electronics.

Liu, E. Theodoropoulos G.(2014)"Space-time matching algorithms for interest management in distributed virtual environments", ACM TOMACS, vol.24

Liu, R., Wang, L., Lei, J., Wang, Q., \& Ren, Y. (2020). Effects of an immersive virtual reality-based classroom on students' learning performance in science lessons. British Journal of Educational Technology, e13434.

Longo, F., Nicoletti, L., \& Padovano, A. (2018). An interactive, interoperable and ubiquitous mixed 
reality application for a smart learning experience. International Journal of Simulation and Process Modelling, 13(6), 589-603.

Lu, Y., \& Xu, X. (2018). Resource virtualization: a core technology for developing cyber-physical production systems. Journal of Manufacturing Systems, 47, 128-140.

Mastli, M., \& Zhang, J. (2017). Interactive highway construction simulation using game engine and virtual reality for education and training purpose. In Computing in Civil Engineering 2017 (pp. 399406).

Mi, L., Zhang, W., Chen, C. P., Zhou, Y., Li, Y., Yu, B., \& Maitlo, N. (2018, May). A retinal-projection-based near-eye display for virtual reality. In Digital Optics for Immersive Displays (Vol. 10676, p. 106761C). International Society for Optics and Photonics.

Mourtzis, D., Zogopoulos, V., \& Vlachou, E. (2017). Augmented reality application to support remote maintenance as a service in the robotics industry. Procedia Cirp, 63(2017), 46-51.

Muhanna, M. A. (2015) "Virtual reality and the CAVE: Taxonomy, interaction challenges and research directions", Journal of King Saud UniversityComputer and Information Sciences, 27(3), 344361

Muñoz-Saavedra, L., Miró-Amarante, L., \& Domínguez-Morales, M. (2020). Augmented and Virtual Reality Evolution and Future Tendency. Applied Sciences, 10(1), 322.

Oberhauser, M., Dreyer, D., Mamessier, S., Convard, T., Bandow, D., \& Hillebrand, A. (2015, August). Bridging the gap between desktop research and full flight simulators for human factors research. In International Conference on Engineering Psychology and Cognitive Ergonomics (pp. 460471). Springer, Cham.

Papachristos, N. M., Vrellis, I., \& Mikropoulos, T. A. (2017, July). A comparison between oculus rift and a low-cost smartphone VR headset: immersive user experience and learning. In 2017 IEEE 17th International Conference on Advanced Learning Technologies (ICALT) (pp. 477-481). IEEE.

Park, J., Chen, J., \& Cho, Y. K. (2017). Self-corrective knowledge-based hybrid tracking system using BIM and multimodal sensors. Advanced Engineering Informatics, 32, 126-138.

Pence, H. E. (2010). Smartphones, smart objects, and augmented reality. The Reference Librarian, 52(12), 136-145.

Pollini, L., Innocenti, M., \& Petrone, A. (2008). Novel motion platform for flight simulators using an anthropomorphic robot. Journal of Aerospace Computing, Information, and Communication, 5(7), 175-196.
Puig, A., Rodríguez, I., Arcos, J. L., Rodríguez-Aguilar, J. A., Cebrián, S., Bogdanovych, A., ... \& Piqué, R. (2020). Lessons learned from supplementing archaeological museum exhibitions with virtual reality. Virtual Reality, 24(2), 343-358.

Qi, Q., \& Tao, F. (2018). Digital twin and big data towards smart manufacturing and industry 4.0: 360 degree comparison. Ieee Access, 6, 3585-3593.

Sagayam, K. M., \& Hemanth, D. J. (2017). Hand posture and gesture recognition techniques for virtual reality applications: a survey. Virtual Reality, 21(2), 91-107.

Schroeder, J. A. (1999). Helicopter flight simulation motion platform requirements, NASA Technical Report, Ames Research Center, Moffett Field, California

Shin, J., An, G., Park, J. S., Baek, S. J., \& Lee, K. (2016). Application of precise indoor position tracking to immersive virtual reality with translational movement support. Multimedia tools and applications, 75(20), 12331-12350.

Steed, A., \& Julier, S. (2013, March). Design and implementation of an immersive virtual reality system based on a smartphone platform. In 2013 IEEE Symposium on 3D User Interfaces (3DUI) (pp. 43-46). IEEE.

Thöndel, E. (2010). Electric motion platform for use in simulation technology-design and optimal control of a linear electromechanical actuator. In Proceedings of the World Congress on Engineering and Computer Science (Vol. 2).

VanderVliet, G. (1992). The Test, Evaluation, Development, and Use of a Manned Flight Simulator to Support Navy Developmental Testing of the V-22 Osprey (No. 921978). SAE Technical Paper.

Wang, L. J., Hu, Q. N., \& Shu, H. (2011). Research on Technologies of Machining Virtual Experiment. Donghua Daxue Xuebao(Ziran Ban), 37(4), 462466.

Wang, L., Groves, P. D., \& Ziebart, M. K. (2012). Multiconstellation GNSS performance evaluation for urban canyons using large virtual reality city models. The Journal of Navigation, 65(3), 459-476.

Whisker, V. E., Baratta, A. J., Yerrapathruni, S., Messner, J. I., Shaw, T. S., Warren, M. E., Rotthoff E.S., Winters J.W., Clelland J.A. \& Johnson, F. T. (2003). "Using immersive virtual environments to develop and visualize construction schedules for advanced nuclear power plants". In Proceedings of ICAPP Vol. 3, pp. 4-7

Zeigler, B. P., \& Lee, J. S. (1998, August). Theory of quantized systems: formal basis for DEVS/HLA distributed simulation environment. In Enabling Technology for Simulation Science II (Vol. 3369, pp. 49-58). International Society for Optics and 
Photonics.

Zhang, Y., Fei, Y., Xu, L., \& Sun, G. (2015, July). MicroIMU-based motion tracking system for virtual training. In 2015 34th Chinese Control Conference (CCC) (pp. 7753-7758). IEEE. 